\title{
Trazabilidad para la cadena de suministro agrícola desde un enfoque informático
}

\section{Traceability for the agricultural supply chain from a computer approach}

OCHOA-ORNELAS, Raquel $\dagger^{*}$, FAJARDO-DELGADO, Daniel, CERVANTES-ZAMBRANO, Francisco y GUDIÑO-VENEGAS, René

Tecnológico Nacional de México / Instituto Tecnológico de Ciudad Guzmán

ID $1^{\text {er }}$ Autor: Raquel, Ochoa Ornelas / ORC ID: 0000-003-1824-5789, Researcher ID Thomson: S-4687-2018, arXiv Author ID: RaquelOchoa, CVU CONACYT ID: 668976

ID $1^{\text {er }}$ Coautor: Daniel, Fajardo Delgado / ORC ID: 0000-0001-8215-5927, Researcher ID Thomson: B-5078-2014, arXiv Author ID: dfajardod, CVU CONACYT ID: 101301

ID $2^{\text {er }}$ Coautor: Francisco, Cervantes Zambrano / ORC ID: 0000-0001-7320-4758, Researcher ID Thomson: S-4951-2018, CVU CONACYT ID: 648147

ID $3{ }^{\text {er }}$ Coautor: René, Gudiño Venegas / ORC ID: 0000-0002-1622-5779, Researcher ID Thomson: ABF-3957-2020, arXiv Author ID: renegv, CVU CONACYT ID: 827306

DOI: $10.35429 / J T I P .2020 .11 .4 .1 .7$

Recibido 30 de Agosto, 2020; Aceptado 09 Diciembre, 2020

\begin{abstract}
Resumen
La trazabilidad es indispensable en el sector agrícola como requisito en la cadena de suministro, ya que genera información valiosa desde el cultivo hasta la comercialización. En este artículo se presenta un sistema que concentra información de las diferentes actividades que se realizan en las huertas de cultivos de aguacate. La plataforma incluye el monitoreo de las actividades mediante la conexión a una base de datos en la nube que permite acceder a los diferentes procesos que se registran a través de una aplicación de escritorio y una página Web. El sistema también controla la información que se genera en la bitácora de mantenimiento, así como la supervisión de las huertas a través de vehículos aéreos no tripulados que actualmente son esenciales para mejorar las condiciones de los cultivos en las huertas. De esta manera, se controlan las labores de mantenimiento con eficiencia, cuidando el medio ambiente a través del uso adecuado de fertilizantes y otros químicos que se emplean en el campo agrícola.
\end{abstract}

Trazabilidad, Bases de datos, huertas, Mantenimiento, bitácora

\begin{abstract}
Traceability is essential in the agricultural sector as a requirement in the supply chain, as it generates valuable information from cultivation to commercialization. In this paper we present a system that concentrates information on the different activities carried out in avocado orchards. The platform includes monitoring activities by connecting to a cloud database that allows access to the different processes that are recorded through a desktop application and a Web page. The system also controls the information that is generated in the maintenance $\log$, as well as the supervision of the orchards through unmanned aerial vehicles that are currently essential to improve the conditions of the crops in the orchards. In this way, maintenance work is controlled efficiently, taking care of the environment through the proper use of fertilizers and other chemicals used in the agricultural field.
\end{abstract}

Traceability, databases, orchards, maintenance, log

Citación: OCHOA-ORNELAS, Raquel, FAJARDO-DELGADO, Daniel, CERVANTES-ZAMBRANO, Francisco y GUDIÑO-VENEGAS, René. Trazabilidad para la cadena de suministro agrícola desde un enfoque informático. Revista de Tecnologías en Procesos Industriales. 2020. 4-11: 1-7

\footnotetext{
* Correspondencia del Autor (raqueoo@itcg.edu,mx)

$\dagger$ Investigador contribuyendo como primer autor.
} 


\section{Introducción}

La competencia a nivel mundial, así como la exigencia de los consumidores de alimentos y bebidas, exigen actualmente que la fabricación y producción de artículos sea más confiable y trasparente para garantizar la inocuidad y seguridad. La trazabilidad de lo que se produce o cultiva es necesaria también desde el punto de vista logístico, para lograr tener un control detallado del producto, desde su entrada como materia prima hasta su procesamiento y posterior almacenamiento para la distribución a través de toda la cadena de suministro (Coello \& González, 2019).

En este artículo se presenta una propuesta de un sistema de información local que se complementa con una página Web, ambas con conexión a una base de datos en la nube, que permite supervisar las actividades de mantenimiento, cortes, compras, pedidos, ventas y cobros en la huerta aguacatera "Ocote Cuate", ubicada en la zona sur del estado de Jalisco.

\section{Antecedentes}

$\mathrm{Al}$ presentarse en Europa una crisis sanitaria en 1996 por el impacto de la enfermedad de las Vacas Locas, se genera el tema de trazabilidad. Actualmente, la trazabilidad es indispensable para los clientes consumidores de alimentos, por lo que se han establecido reglas para implementarla de manera obligada. La trazabilidad se aplica a varios sectores entre los que destacan los productos alimentarios, agrícolas, agropecuarios y de construcción (Dionicio Reynoso, 2017).

Los sistemas de trazabilidad facilitan la planificación y organización de la información de un producto a lo largo de la cadena de suministro, recorriendo el proceso en cada uno de sus elementos desde que ingresan a la organización hasta que salen, realizando balances de existencias en palé o cajas con los valores que detecta el sistema, teniendo además en cuenta identificados la ubicación de los productos (Huertas \& Rincón, 2017). La cadena de suministro agroalimentaria se integra a través del suministro de insumos, producción, poscosecha, almacenamiento, procesamiento, comercialización, distribución y consumo, registrando cualquier detalle relacionado con información de las siembras, registro de irrigación, fertilización y control de plagas.
Así como también el control de lotes por cosecha que incluye la parcela o huerta en donde fue producido (Dionicio Reynoso, 2017). Dionicio Reynoso (2017) mencionan como requisitos de un sistema de trazabilidad, el intercambio de información entre actores de la cadena, definir criterios para agrupar productos, identificar lote, uso de tecnología y manejo de registros, así como respetar normas de seguridad alimentaria.

De esta manera, las Tecnologías de Información y Computación (TIC) permiten comparar precios e interactuar con empresas contribuyendo a la mejora de los productos y servicios fortaleciendo la competencia en los mercados.

Actualmente en la agricultura también se emplean vehículos aéreos no tripulados para realizar una supervisión visual de las explotaciones agrícolas por medio del análisis de imágenes y videos que son capturados durante el vuelo. Estos artefactos proporcionan información confiable sobre el estado de las cosechas sin tener que recorrer personalmente los campos de cultivo (Telefónica, 2015).

\section{Objetivos}

\subsection{Objetivo general:}

Desarrollar un sistema de información de trazabilidad para la cadena de suministro agrícola en las huertas de aguacate.

\subsection{Objetivos específicos:}

- Disponer de una bitácora de mantenimiento de las huertas.

- Registrar la cosecha o producción de cada una las huertas.

- Registrar las diferentes adquisiciones mediante un módulo de compras.

- Llevar el seguimiento de los pedidos de los clientes.

- Tener un control sobre las ventas realizadas.

- Controlar la cobranza de las ventas efectuadas.

Obtener diferentes reportes y estadísticas de seguimiento. 


\section{Marco teórico}

\subsection{Scrum y UML}

Entre las metodologías más importantes de desarrollo ágil se considera Scrum, donde se refleja una participación activa del cliente a través de la gestión de las actividades del proyecto. El ciclo de vida iterativo e incremental de UML (Lenguaje Unificado de Modelado) interviene al describir el modelo de un sistema que incluyen procesos del negocio, funciones del sistema y esquemas de bases de datos. En los proyectos iterativos e incrementales los requerimientos no son especificados al inicio del proyecto, sino que evolucionan por medio de una retroalimentación constante entre el desarrollador y el cliente, por lo que el cliente participa de una manera activa obteniendo finalmente un producto adaptado a sus requerimientos (Fuentes, 2015).

\subsection{Plataforma .NET}

Santos (2018) describe al .NET como un conjunto de herramientas y tecnologías que ofrece Microsoft para desarrollar aplicaciones en Windows, Web, smartphones, tabletas y sistemas embebidos. Estas aplicaciones se desarrollan a través de un framework .NET que incluye varios componentes. El framework .NET está formado por CLR (Common Language Runtime, por sus siglas en inglés), DLR (Dynamic Language Runtime, por sus siglas en inglés) y la Base Class Library (biblioteca de clases básicas).

\subsection{Ajax}

Ajax (Asynchrounous JavaScript and XML) surgió en 2005 como la conjunción de tecnologías independientes aplicadas al desarrollo Web. Usa el objeto "XMLHttpRequest", que permite comunicar el cliente y el servidor de manera asíncrona transformando las páginas Web en interactivas.

La comunicación asíncrona recupera información de forma oculta para el usuario, modifica peticiones HTTP por peticiones JavaScript, interactuando de una manera rápida y adecuada (Barrera, 2018).

\subsection{PHP}

PHP es un lenguaje de programación Web que soporta diferentes motores de bases de datos tales como Microsoft SQL Server, IBM DB2. PostgreSQL, MySQL y Oracle. Algunas nuevas características de PHP 7 incluyen las declaraciones de tipo escalar: coercitivo (por defecto) y estricto. Además, considera nuevas características de sintaxis mejorando el código y agrupando declaraciones de importación. También aporta nuevas funciones y clases de excepción, así como optimizaciones de alto rendimiento (PHP, 2020).

\subsection{MySQL}

MySQL es un gestor de base de datos empleado en el desarrollo Web para implementar aplicaciones nativas en la nube. Fue desarrollado, administrado y respaldado por el equipo de MySQL AB, posteriormente fue adquirido por Sun Microsystem y finalmente por Oracle Corporation en 2010. Se desarrolló con base en los lenguajes $\mathrm{C}$ y $\mathrm{C}++$, y se ejecuta tanto en Linux como en Windows Server, siendo los sistemas GNU/Linux donde principalmente optimiza su funcionamiento. Utiliza motores como MyISAM o InnoDB. Organizaciones importantes en el mundo, incluidas Facebook, Google, Adobe, Alcatel Lucent y Zappos, Youtube y Twitter utilizan MySQL en sus sitios Web. MySQL 8.0 actualmente es la última versión estable disponible (MySQL, 2020).

\subsection{Bootstrap}

Barrera (2018) define a Bootstrap como un framework de librerías de software libre que permite crear aplicaciones Web adaptables o responsivas, minimizando además tiempos de desarrollo. Bootstrap es compatible con sistemas operativos de móviles. Es adaptable a varios navegadores de Internet. Está desarrollado bajo los últimos estándares de HTML5, CSS3 y jQuery. Bootstrap se basa en un sistema de rejillas para posicionar elementos. Para su implementación es necesario incorporar a la aplicación en desarrollo un archivo CSS y otro en JavaScript. 


\subsection{UAV}

Los drones o vehículos aéreos no tripulados (UAV) están asociados con aplicaciones de defensa y en actividades civiles, incluidos el transporte, las comunicaciones, la agricultura, la mitigación de desastres y la conservación del medio ambiente. Este tipo de dispositivos presenta grandes desafíos científicos y técnicos por el costo energético de mantenerse en vuelo continuo, así como la inteligencia perceptiva en entornos complejos. (Floreano \& Wood, 2015).

López-Granados (2013) describe las ventajas de los drones con relación a los aviones convencionales o a las imágenes de satélite:

Trabajan en autonomía programando vuelos en momentos críticos bajo cualquier pronóstico meteorológico.

- Pueden incorporar sensores con distinto rango espectral.

- Generan imágenes de alta resolución.

De la misma manera, considera cuatro fases en la gestión localizada:

Monitorización: detecta variables
(hongos y maleza).
- $\begin{aligned} & \text { Decisiones: elabora mapa de } \\ & \text { tratamientos y plan de actuación. }\end{aligned}$
- Actuación: ejecuta acciones en
localización.
Evaluación: analiza y evalúa la
rentabilidad tanto económica como
ambiental de las operaciones realizadas
llevando un registro en una bitácora.

\section{Metodología}

El sistema de información comprende una aplicación de escritorio que es instalada localmente y una página Web, ambas conectadas a una misma base de datos en la nube. Se aplicó la metodología de Scrum que es un marco de programación ágil basado en una estrategia de desarrollo iterativo e incremental que asegura la calidad del producto.

Del análisis de los requerimientos se determinó que todos los sistemas de trazabilidad deben reunir ciertos requisitos:
- Intercambio de información y colaboración entre cliente y proveedor.

- $\quad$ Relación entre los actores de la cadena.

- Definir criterios para agrupar productos (lotes, unitarios, lotes multiproducto, caja o palé).

- Sistema de codificación (código de barras).

- Establecer registros y documentación necesarios.

- Herramientas para recopilar información (Lectores de código de barras).

- $\quad$ Manejo de registro y almacenamiento.

- Cumplir con regularizaciones de seguridad sanitaria.

Así también, los diferentes procesos de negocio relacionados a los sistemas de trazabilidad agrícola incluyen la captura de información, registrar y almacenar información y suministrar información a las partes interesadas.

Los procesos de negocio relacionados con la trazabilidad de la cadena de suministro agrícola son:

1. Cultivo: considera tipo de semilla o cepa utilizado, humedad de la tierra donde se sembró, tipo de abono o fertilizante utilizado, plaguicida y cantidad de agua.

2. Producción: se refiere al corte de la fruta de aguacate y empacado del producto en cajas.

3. Almacenamiento: tipo de almacén utilizado, temperatura y agrupación de los productos.

4. Aprovisionamiento: disponer de un registro detallado de compras de insumos.

5. Distribución y ventas: registrar vehículos que fueron utilizados para la distribución del producto, temperatura y tiempo de transporte, así como si se sufrió de algún tipo de percance. También se almacena la información relacionada con los clientes, ventas y el precio de la venta del producto.

6. Logística: tiene que ver en toda la cadena de suministro desde el cultivo, mantenimiento de la huerta, producción transporte y venta. 
En el análisis se desarrollaron diferentes diagramas en UML que permitieron capturar los requisitos funcionales y no funcionales a través de una serie de entrevistas con los propietarios de las huertas. Posteriormente, las tareas principales que se llevan a cabo fueron clasificadas en procesos, identificando entidades externas y almacenes de datos.

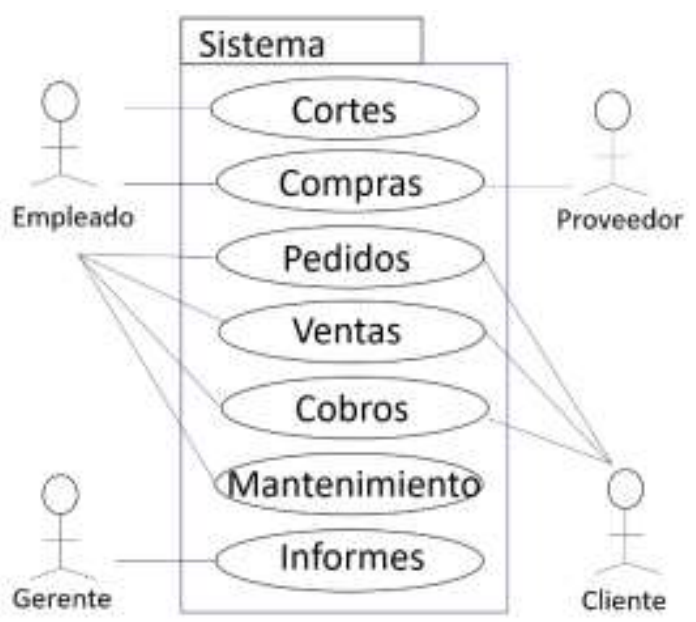

Figura 1 Diagrama de casos de uso general

Durante el diseño, se definió la base de datos y tablas en MySQL así como los roles de los diferentes usuarios, interfaces y la estructura de los informes que genera el sistema. En la Figura 2 se presenta el diagrama de despliegue que comprende la aplicación de escritorio desarrollada en Visual Basic y la aplicación Web en el rol de empresa desarrollada en PHP con conexión a MySQL, así como la aplicación Web en el rol de clientes para rastrear pedidos y pagos pendientes.

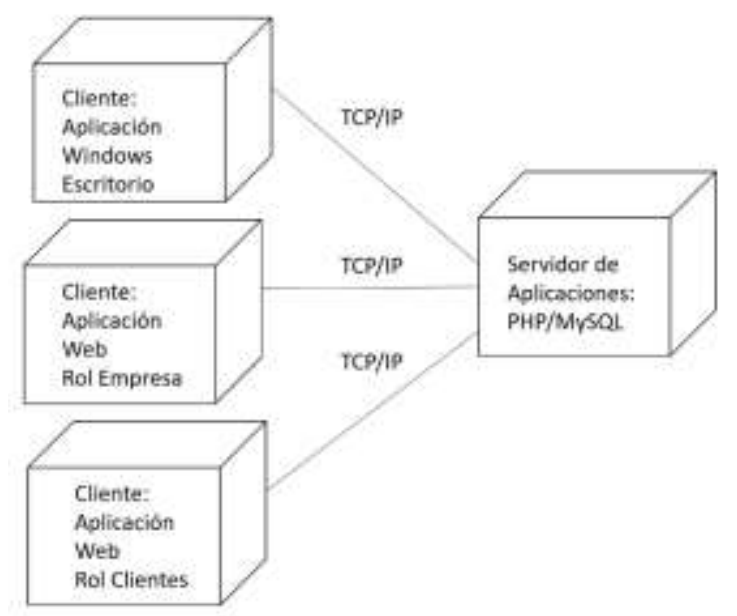

Figura 2 Diagrama de despliegue

Al final se realizaron pruebas unitarias y pruebas integrales donde se detectaron algunos defectos que posteriormente fueron corregidos.
Durante la implantación se capacitaron a los usuarios, así como también se llevó a cabo un inventario de todos los suministros $\mathrm{y}$ herramientas, capturando catálogos y movimientos. Posteriormente se comprobaron los resultados en forma paralela hasta liberar finalmente el sistema para iniciar con la transición.

\section{Resultados}

En la Figura 3 se presenta el menú de los catálogos (clientes, proveedores, empleados, tipos de productos, productos a la venta, tipo de materia prima, materia prima, actividades de postcosecha y huertos).

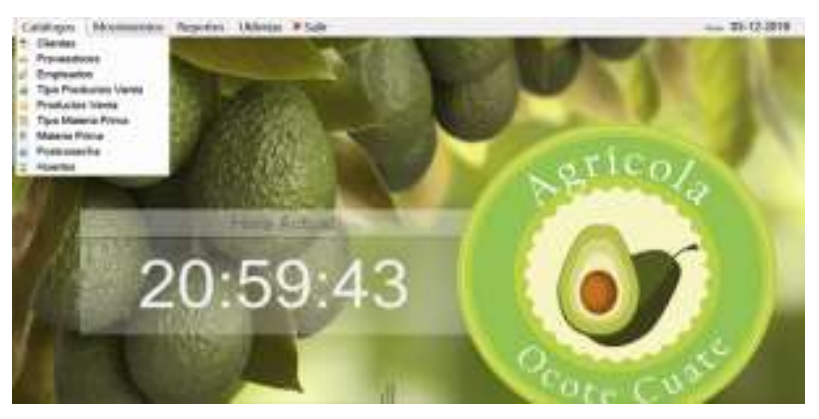

Figura 3 Menú de catálogos

En la Figura 4 se presenta la interfaz del catálogo de clientes.

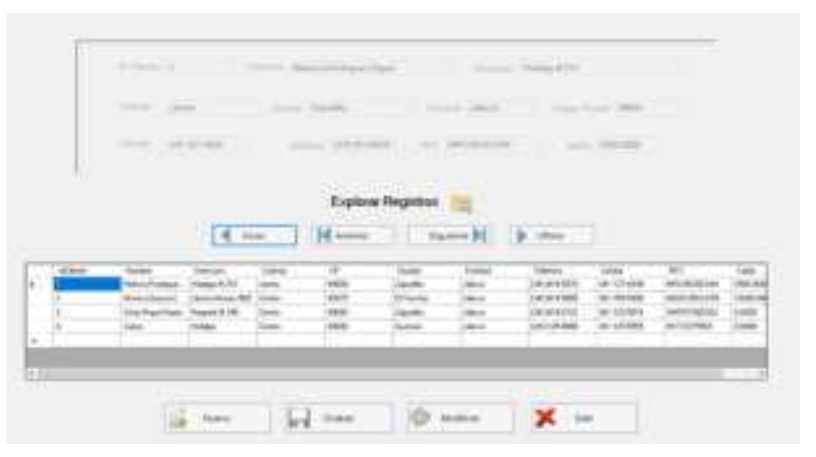

Figura 4 Interfaz de clientes

En la Figura 5 se presenta la interfaz de catálogo de productos.

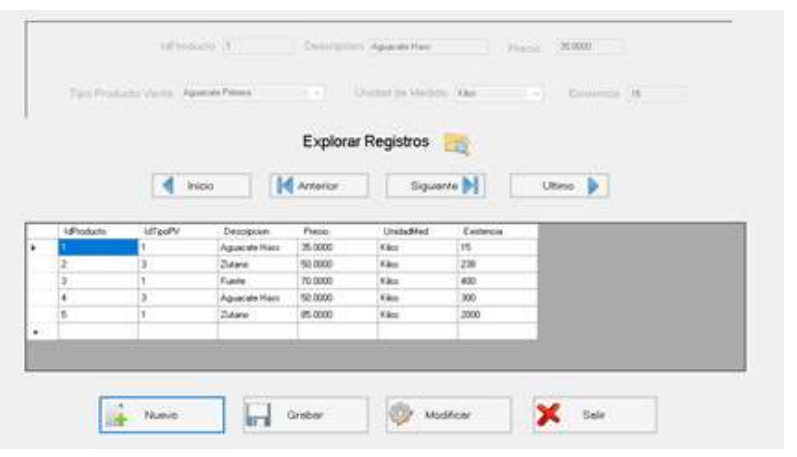

Figura 5 Interfaz de productos

OCHOA-ORNELAS, Raquel, FAJARDO-DELGADO, Daniel, CERVANTES-ZAMBRANO, Francisco y GUDIÑO-VENEGAS, René. Trazabilidad para la cadena de suministro agrícola desde un enfoque informático. Revista de Tecnologías en Procesos Industriales. 2020 
En la Figura 6 se presenta la interfaz de catálogo de materia prima.

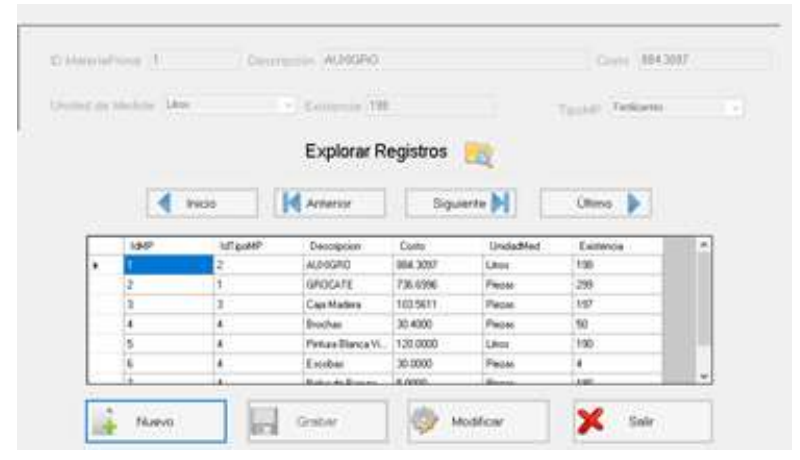

Figura 6 Interfaz de materia prima

En la Figura 7 se presentan las opciones de movimientos.

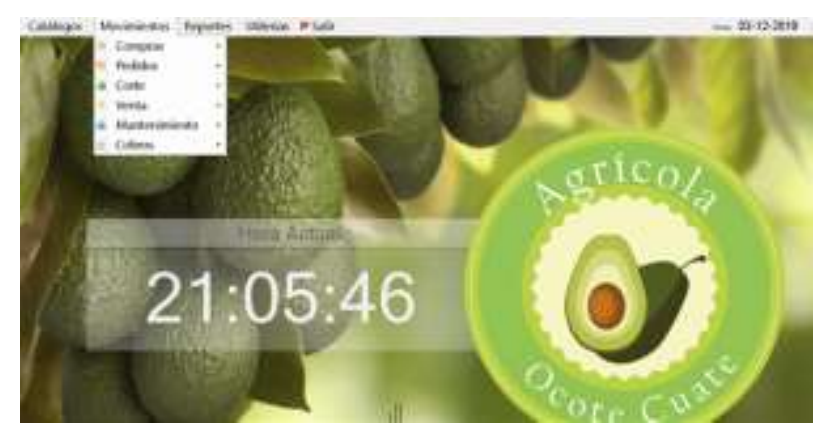

Figura 7 Opciones de movimientos

En la Figura 8 se presenta la interfaz para el registro de compras.

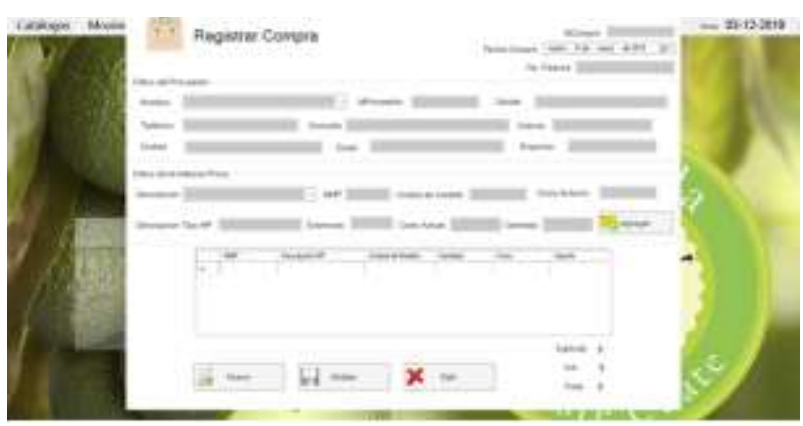

Figura 8 Interfaz de compras

En la Figura 9 se presenta la interfaz para el registro de mantenimiento.

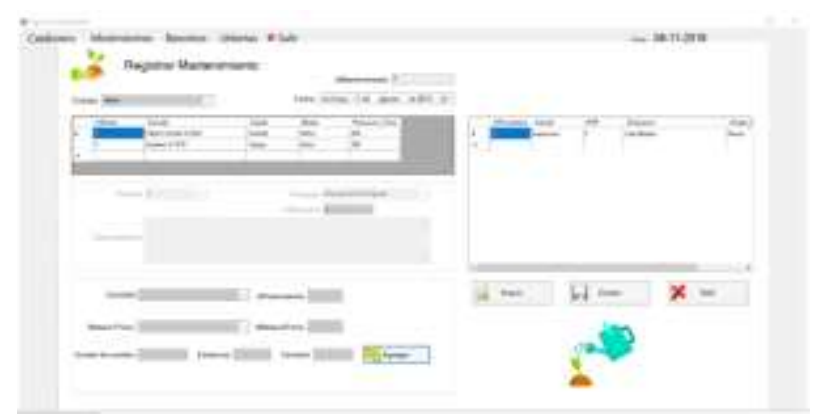

Figura 9 Interfaz para el registro de mantenimiento

ISSN 2523-6822

ECORFAN® Todos los derechos reservados
En la Figura 10 se muestra el reporte de corte en un periodo.

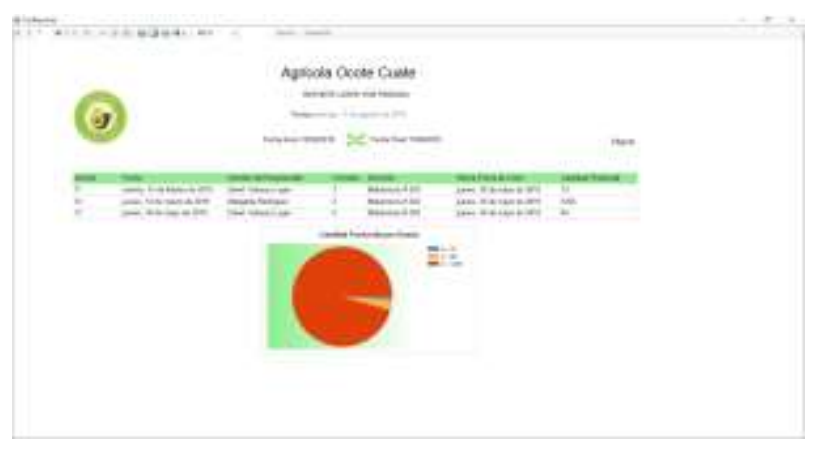

Figura 10 Reporte de corte en un periodo

En la Figura 11 se presenta la aplicación Web en el rol del cliente para consultar pedidos.

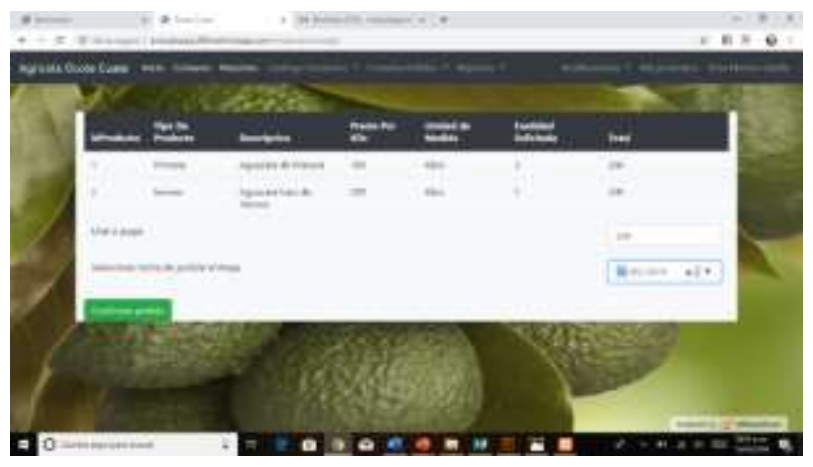

Figura 11 Consulta de pedidos por el cliente

En la Figura 12 se muestra el dron utilizado para registrar las fotografías durante los recorridos en que se realiza mantenimiento en la huerta.

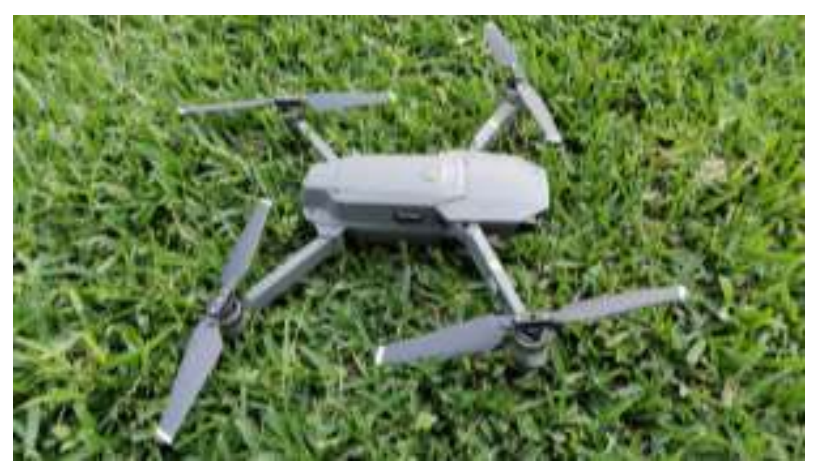

Figura 12 Dron para recorridos en huertas

En la Figura 13 se muestran imágenes obtenidas por el dron durante los recorridos. 


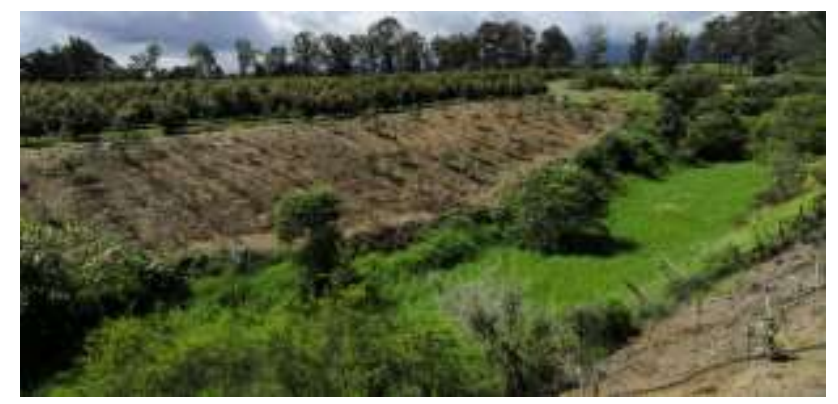

Figura 13 Recorridos en huertas

\section{Conclusiones}

Uno de los mayores beneficios de la trazabilidad es la contribución que realiza la tecnología aportando sistemas de información que favorecen a la sostenibilidad agrícola, incrementando la calidad de los sistemas de producción de alimentos, otorgando mayor fiabilidad y trasparencia.

Al incorporar los sistemas de información en las tareas del campo se obtienen ventajas competitivas contribuyendo a incrementar la productividad en el control de las actividades. Así también, se protege al medio ambiente al tener un control eficiente en la aplicación de fertilizantes y plaguicidas.

El primer autor agradece al Tecnológico Nacional de México el apoyo otorgado para realizar este proyecto de investigación registrado con número 6216.19-P.

\section{Referencias}

Barrera, C. E. (2018). Desarrollo de un Sistema Web Multiplataforma de Gestión de Selección de Personal para el área de Talento Humano, utilizando el Framework Bootstrap (Bachelor's thesis, Universidad del Azuay). Obtenida el 10 de noviembre de 2019, de la página electrónica: http://dspace.uazuay.edu.ec/handle/datos/7939

Coello Machado, N. I., \& González Cabrera, E. (2019, June). Concepciones teóricas de la trazabilidad en la cadena de suministros de alimentos y bebidas.

Dionicio Reynoso, C. A. (2017). Propuesta de un sistema de trazabilidad para la cadena de suministro agrícola en un contexto de colaboración. Obtenida el 8 de noviembre de 2019, de la página electrónica: https://riunet.upv.es/handle/10251/90728
Floreano, D., \& Wood, R. J. (2015). Science, technology and the future of small autonomous drones. Nature, 521(7553), 460-466.

Fuentes, J. R. L. (2015). Desarrollo de Software ÁGIL: Extreme Programming y Scrum. IT Campus Academy.

Huertas Díaz, D. M., \& Rincón Vargas, H. V. (2017). Diseño de un sistema de trazabilidad de los materiales de consumo en el almacén de la sede judicial Hernando Morales Molina de Bogotá.

López-Granados, F. (2013). Uso de Vehículos Aéreos no tripulados (UAV) para la evaluación de la producción agraria [versión electrónica]. ambienta, (105), 40-52.

MySQL. (2020). Mysql Database Service. Obtenido el 14 de noviembre de 2020, de la página electrónica: https://www.mysql.com/

PHP (2020). Nuevas características. Obtenido el 14 de noviembre de 2020, de la página electrónica:

https://www.php.net/manual/es/migration70.ne w-features.php

Santos, G. A. D. (2018). Análisis comparativo de los ide's de desarrollo xamarin visual studio vs android studio aplicado al sistema de notificación de actividades de la UNACH, 2017 (Bachelor's thesis, Riobamba, Universidad Nacional de Chimborazo). Obtenida el 8 de noviembre de 2019, de la página electrónica: http://190.15.135.60/handle/51000/4688

Telefónica, F. (2015). El trabajo en un mundo de sistemas inteligentes. Fundación Telefónica. Barcelona: Editorial Ariel, S.A. 